\title{
PROFILI EVOLUTIVI DEI PIÙ RECENTI SVILUPPI DELLA GIURISPRUDENZA CONSTITUZIONALE ITALIANA CON RIFERIMENTO ALLA CONVENZIONE EUROPEA DEI DIRITTI DELL'UOMO
}

\section{PERFIS DA EVOLUÇÃO DOS DESENVOLVIMENTOS MAIS RECENTES NA LEI CONSTITUCIONAL ITALIANO COM REFERENCIA À CONVENÇÃO EUROPÉIA DE DIREITOS HUMANOS \\ Francesco Bilancia}

Università degli Studi "G d'Annunzio" di Chieti-Pescara - Itália

Riassunto: L'obiettivo era quello di analizzare giurisprudenza costituzionale italiana per quanto riguarda l'uso di accordi internazionali per la protezione dei diritti umani, dato che tali accordi è solo interferiscono con la formattazione dello Stato stesso. II processo di interazione tra i vari documenti costituzionali dello Stato, e tra le lettere di tutela dei diritti fondamentali queste ultime e internazionali, è stato lentamente, a volte un po 'incerto, pieno di battute d'arresto reali, così l'articolo ha considerato l'analisi antinomie derivante dall'interazione, non terra sempre comune tra legge italiana e le norme della CEDU. La ricerca è guidata da alcune recenti decisioni della Corte costituzionale italiana, facendo riferimento alla Convenzione europea dei diritti dell'uomo (CEDU) e la giurisprudenza della Corte europea dei diritti dell'uomo.

Parole-chiave: diritti umani - CEDU - antinomie - diritti interno.

Resumo: O objetivo do trabalho foi analisar a jurisprudência constitucional italiana no que diz respeito à utilização de acordos internacionais para proteger os direitos humanos, haja vista que tais acordos acabam for interferir na formatação do próprio Estado. O processo de interação entre os vários documentos constitucionais do Estado, e entre estes e as cartas internacionais de proteção dos direitos fundamentais, tem andado a passos lentos, e as vezes um tanto incerto, cheio de contratempos reais, assim o artigo debruçou-se sobre 
a análise de antinomias que surgem do convívio, nem sempre pacífico, entre a legislação italiana e as normas de CEDH.A pesquisa norteia-se a partir de algumas decisões recentes do Tribunal Constitucional italiano, referindo-se à Convenção Européia de Direitos Humanos (CEDH) e a jurisprudência do Tribunal Europeu dos Direitos do Homem.

Palavras-chave: direitos humanos - CEDH - antinomia - direito interno.

\section{Premessa}

Questo breve scritto intende fornire un quadro d'insieme dei più recenti sviluppi della giurisprudenza costituzionale italiana con riferimento all'uso degli accordi internazionali di protezione dei diritti umani, che opera attraverso un "processo di grandiose proporzioni, il quale investe il futuro stesso dello Stato: non di questo o quello Stato, ma - se così può dirsi - della forma-Stato". Questo processo, destinato a svolgersi in un indefinibile arco di tempo ma costantemente sostenuto dalla più attenta giurisprudenza, non avrebbe - non ha - potuto "non investire il destino della stessa (...) Costituzione ${ }^{1 "}$. La lunga e complicata evoluzione del processo di "interazione" tra i diversi documenti costituzionali statali e tra questi e le Carte internazionali di protezione dei diritti fondamentali si è spesso caratterizzato per un cammino di piccoli passi, di fasi di integrazione a volte più intense, a volte più incerte, senza escludere vere e proprie battute d'arresto, ma comunque qualificato ed arricchito da importanti episodi giurisprudenziali di cui, momento per momento, la dottrina ha preteso di ricostruire la fotografia di sintesi, nell'incessante vano tentativo di ridurre la complessità a sistema. Non è, è bene dirlo subito, l'intenzione di queste brevi note che traggono, piuttosto, spunto da alcune più recenti pronunce della Corte costituzionale italiana, riferite alla Convenzione ed alla giurisprudenza della Corte europea dei diritti dell'Uomo (CEDU), riconducibili al percorso giurisprudenziale

\footnotetext{
${ }^{1}$ A. D'Atena, Costituzionalismo moderno e tutela dei diritti fondamentali, in A. D'Atena, P. Grossi (a cura di), Tutela dei diritti fondamentali e costituzionalismo multilivello tra Europa e Stati nazionali, Milano, 2004, $33 \mathrm{~s}$. 
avviato a partire dalle sentenze nn. 348 e 349 del $2007^{2}$. Come è noto ormai la Corte costituzionale italiana riconduce il contrasto tra una norma interna ed una norma della Convenzione europea dei diritti dell'uomo alla violazione mediata dell'art. 117, comma 1, Cost., di cui la stessa Corte costituzionale dovrà essere investita nel caso in cui il giudice interno non sia in condizione di risolvere l'antinomia per via di interpretazione conforme. A giudizio della Corte resta, infatti, preclusa al giudice di merito la strada dell'applicazione diretta della norma CEDU mediante la contestuale disapplicazione della norma interna incompatibile ${ }^{3}$, ritenendo non assimilabile tale sistema di garanzie allo schema di adattamento del diritto interno al diritto comunitario e dell'UE ${ }^{4}$. Piuttosto, a giudizio della Corte

\footnotetext{
${ }^{2}$ In Giur.cost., 2007, risp. 3475 ss. e 3536 ss., con i commenti di C. Pinelli, Sul trattamento giurisdizionale della CEDU e delle leggi con essa configgenti, 3518 ss.; A. Moscarini, Indennità di espropriazione e valore di mercato del bene: un passo avanti (ed uno indietro) della Consulta nella costruzione del patrimonio costituzionale europeo, 3525 ss.; M. Cartabia, Le sentenze "gemelle»: diritti fondamentali, fonti, giudici, 3564 ss.; A. Guazzarotti, La Corte e la CEDU: il problematico confronto di standard di tutela alla luce dell'art. 117, comma 1, Cost., 3574 ss.; V. Sciarabba, Nuovi punti fermi (e questioni aperte) nei rapporti tra fonti e corti nazionali e internazionali, 3579 ss. ma si vedano, altresì, M. Luciani, Alcuni interrogativi sul nuovo corso della giurisprudenza costituzionale in ordine ai rapporti fra diritto italiano e diritto internazionale, in Corriere giuridico, 2/2008, 201 ss.; C. Focarelli, Lezioni di diritto internazionale, I, Padova, 2008, 237 ss.; F. Sorrentino, Apologia delle "sentenze gemelle" (brevi note a margine delle sentenze nn. 348 349/2007 della Corte costituzionale, in Dir.soc., 2009, 213 ss.; G. Repetto, Diritti fondamentali e sovranità nello stato costituzionale chiuso. Prime osservazioni critiche sulla sentenza n. $349 \mathrm{del}$ 2007 della Corte costituzionale, in Giur.it., 2008, 309 ss.; B. Conforti, La Corte costituzionale e gli obblighi internazionali dello Stato in tema di espropriazione, ivi, 569 ss.; R. Calvano, La Corte costituzionale e la CEDU nella sentenza n. 348 del 2007: orgoglio e pregiudizio, ivi, 573 ss.; F. Angelini, L'incidenza della CEDU nell'ordinamento italiano alla luce di due recenti pronunce della Corte costituzionale, in II diritto dell'UE, 2008, 487 ss.; M.E. Bartoloni, Un nuovo orientamento della Corte costituzionale sui rapporti fra ordinamento comunitario e ordinamento italiano?, ivi, 511 ss.; M. Savino, Il cammino internazionale della Corte costituzionale dopo le sentenze n. 348 e 349 del 2007, in Riv.it.dir.pubbl.com., 2008, 747 ss.; A. Filippini, Il caso Dorigo, la CEDU e la Corte costituzionale: l'effettività della tutela dei diritti dopo le sentenze 348 e 349 del 2007, in www.costituzionalismo.it, 2/2008; ed i commenti di G. Gaja, E. Cannizzaro, M.L. Padelletti e A. Saccucci in Riv.dir.int., 2008, 136 ss. Più in generale sul tema dei rapporti tra CEDU e diritto interno si vedano, infine, i saggi di E. Lamarque, II vincolo alle leggi statali e regionali derivante dagli obblighi internazionali nella giurisprudenza comune, Relazione presentata al Seminario dal titolo "Corte costituzionale, giudici comuni e interpretazioni adeguatrici", Roma, Palazzo della Consulta, 6 novembre 2009, e di R. Niro, Il vento di Strasburgo sui diritti inviolabili: note a margine di qualche pronuncia della Corte europea dei diritti dell'uomo, in Scritti in onore di Alessandro Pace, III, Napoli, 2012, 2253 ss.

${ }^{3} \mathrm{Si}$ vedano, sul punto, le interessanti valutazioni formulate da C. Pinelli, Sul trattamento giurisdizionale, cit., 3519 in relazione alla "incipiente ma potenzialmente inarrestabile tendenza dei giudici comuni a disapplicare le norme legislative configgenti con la CEDU...spostando la prospettazione dell'antinomia dal piano delle fonti...a quello dei diritti da esse rispettivamente riconosciuti". Ancora M. Cartabia, Le sentenze "gemelle», cit., 3566 s., anche per i riferimenti ai sistemi di Common Law. Ma su tali questioni torneremo infra.

${ }^{4}$ Sulla questione si soffermano dettagliatamente B. Conforti, La Corte costituzionale e gli obblighi internazionali, cit., 9 ss.; M. Luciani, Alcuni interrogativi sul nuovo corso, cit., 203 ss.; M. Cartabia, 
costituzionale resta, non solo possibile, ma addirittura necessario verificare, in sede di giudizio di costituzionalità, la specifica compatibilità in concreto della norma CEDU invocata quale parametro, per come interpretata ed applicata dalla Corte di Strasburgo, con le diverse disposizioni costituzionali. Sul piano formale della dottrina costituzionale del sistema delle fonti le disposizioni della CEDU ${ }^{5}$, quindi, in quanto dotate di forza passiva superiore a quella delle norme di legge ordinaria, fungeranno da norme interposte nel giudizio di costituzionalità delle norme interne con esse incompatibili per violazione indiretta dell'art. 117 Cost. Laddove, all'opposto, stante la loro non equiparabilità formale alle disposizioni costituzionali, potrebbe darsi il caso di un giudizio di costituzionalità della legge di recepimento della Convenzione nell'ipotesi di contrasto con altre disposizioni costituzionali; non quindi più soltanto dei principi fondamentali come previsto, secondo la nota dottrina costituzionale dei "controlimiti", con riferimento ai rapporti del diritto interno con le norme di diritto comunitario direttamente applicabili.

\section{I caratteri propri del sistema della Corte europea dei diritti} dell'Uomo in relazione all'ordinamento statale.

Con la successiva sentenza n. 317 del $2009^{6}$ la Corte costituzionale è, sembrata, poi, procedere oltre nella costruzione di un più fitto sistema integrato di garanzie dei diritti fondamentali, in un'ottica di massima espansione degli strumenti di tutela complessivamente riconducibili alla Convenzione europea, nell'interpretazione datane dalla Corte di Strasburgo, per come recepita e ricomposta in un coerente contesto di sistema nel quadro costituzionale interno. Almeno all'esito delle forme di esercizio in concreto dei giudizi di bilanciamento, operati dalla Corte costituzionale italiana, tra il fine della garanzia dei diritti fondamentali perseguito dalla CEDU e i principi costituzionali che si sarebbero altrimenti potuti ritenere con essa in conflitto. E' sembrato, cioè, che la Corte italiana intendesse riformulare il giudizio di bilanciamento tra i valori in conflitto in

Le sentenze "gemelle», cit., 3565 ss.; F. Sorrentino, Apologia delle "sentenze gemelle", cit., 221 ss.; R. Calvano, La Corte costituzionale e la CEDU, cit.; A. Moscarini, Indennità di espropriazione, cit., $3528 \mathrm{~s}$.

${ }^{5}$ Le relative norme interne di recepimento.

${ }^{6}$ Su cui A. Ruggeri, Conferme e novità di fine anno in tema di rapporti tra diritto interno e CEDU (a prima lettura di Corte cost. nn. 311 e 317 del 2009), in Forum dei Quad.cost., dicembre 2009.

REVISTA DO DIREITO UNISC, SANTA CRUZ DO SUL №. 43 | p.03-24 | MAIO-AGO 2014 
una prospettiva che andasse oltre il rischio di non consentirle di assumere, nel singolo caso concreto, la norma CEDU quale parametro di un giudizio di costituzionalità ${ }^{7}$. E' così che nella sentenza n. 317 la Corte ha ritenuto di individuare il parametro di giudizio, non soltanto nell'art. 117, primo comma - "in relazione all'art. 6 CEDU, quale interpretato dalla Corte di Strasburgo" - ma anche negli artt. 24 e 111, primo comma della Costituzione. Nel procedere in concreto alla composizione del giudizio di costituzionalità secondo lo schema formale delineato nella precedente giurisprudenza costituzionale, che viene espressamente richiamata, la Corte ha quindi ritenuto di doversi addentrare nello specifico confronto tra le forme di tutela garantite dall'ordinamento costituzionale italiano e quelle previste dal diritto CEDU, in un'analisi del rispettivo diritto vivente alla ricerca di una possibile comparazione dei rimedi a garanzia dei diritti evocati, sul piano della loro effettività.

Come spero di dimostrare nel prosieguo di queste brevi note, la giurisprudenza costituzionale più recente ha proceduto con più accentuata attenzione a delineare i profili della sostanziale differenza tra il fenomeno della integrazione comunitaria e le ricadute del sistema CEDU nell'ordinamento italiano ${ }^{9}$. II diritto comunitario e dell'UE pervade gli ordinamenti statali dei propri fini e principi, determinando una trasformazione della stessa forma di Stato nazionale funzionalizzando i sistemi giuridici nazionali al perseguimento dei propri obiettivi; la CEDU ed il relativo sistema di protezione dei diritti fondamentali impongono, invece, agli Stati membri che essi garantiscano il rispetto di uno standard minimo di protezione dei diritti e delle libertà senza che ciò debba necessariamente implicare un processo di adattamento normativo alle disposizioni della Convenzione e dei relativi Protocolli addizionali. La norma è che

7 Tale nozione, entrata ormai nell'uso comune e sostenuta da una consolidata prassi giurisprudenziale, qui viene richiamata senza alcuna ulteriore valutazione. Rinvio, comunque, alla ormai risalente e nota ricostruzione sistematica formulata da M. Siclari, Le «norme interposte» nel giudizio di costituzionalità, Padova, 1992.

${ }^{8}$ Nella precedente sentenza n. 311 la Corte costituzionale evoca altresì il potenziale ruolo di parametro rappresentato dall'art. 10, primo comma, Cost., ma soltanto nel caso in cui si raggiunga la dimostrazione che la norma della CEDU sia "ricognitiva di una norma del diritto internazionale generalmente riconosciuta". Tale questione, che pure sarebbe interessante indagare in concreto, non verrà però qui presa in esame. Mi limito perciò a rinviare alla articolata riflessione che all'argomento dedica, con specifico riferimento ai precedenti del 2007, F. Angelini, L'incidenza della CEDU, cit., spec. 496, 502 ss., 509.

${ }^{9}$ Si vedano, altresì, M. Luciani, Alcuni interrogativi, cit., 203 ss.; F. Sorrentino, op.ult.cit., 222 s.

REVISTA DO DIREITO UNISC, SANTA CRUZ DO SUL №. 43 | p.03-24 | MAIO-AGO 2014 
i diritti e le libertà di cui alla CEDU siano garantiti, infatti, in virtù degli stessi sistemi interni di protezione dei diritti di ciascuno Stato, riservandosi la CEDU un controllo diretto soltanto sui singoli casi che gli Stati, o più tipicamente i singoli individui che si pretendano vittime di una violazione della Convenzione, sottopongano alla verifica della Corte di Strasburgo nell'ipotesi residuale in cui tutti gli strumenti di garanzia predisposti da quel sistema giuridico nazionale abbiano, proprio in quello specifico caso, fallito. E che questo sia il meccanismo sostanziale di protezione dei diritti predisposto dal diritto della CEDU lo dimostra la centralità, in quel sistema, della regola del necessario previo esaurimento delle vie di ricorso interne ${ }^{10}$ perché un ricorso contro uno Stato membro possa essere dichiarato ammissibile ${ }^{11}$ dalla Corte di Strasburgo.

Come vedremo in conclusione non è, peraltro, escluso che alcune pronunce della Corte europea dei diritti dell'uomo possano assumere rilevanza al di là del singolo caso concreto, determinando importanti conseguenze in relazione alle forme ed ai mezzi con cui gli Stati saranno chiamati ad ottemperare agli obblighi di esecuzione di tali sentenze. Ciò accade, ad esempio, ogni qual volta la giurisprudenza CEDU consolidi il proprio case-law su ricorrenti fattispecie che rivelino una violazione sistematica della Convenzione da parte di uno Stato membro. Si tratta dei non infrequenti casi in cui a rilevare non sia più una questione di fallimento del sistema interno delle garanzie dei diritti in un singolo caso, ma si appalesi l'evidente incompatibilità di qualche istituto, di una prassi o, più direttamente, della normativa interna con i livelli di protezione dei diritti e delle libertà fondamentali garantiti dalla CEDU, nella lettura datane dalla Corte di Strasburgo. Laddove la violazione sistematica denunciata dal consolidamento della giurisprudenza della Corte europea su casi identici e ripetuti riveli, quindi, un difetto strutturale dell'ordinamento statale al cospetto delle garanzie imposte dal sistema CEDU l'obbligo di adattamento dell'ordinamento interno al paradigma CEDU dovrà assumere la forma non più della riparazione nei confronti del singolo

\footnotetext{
${ }^{10}$ Art. 35 .

${ }^{11}$ Ho dettagliatamente analizzato il sistema CEDU con riferimento ai sistemi giuridici nazionali nel mio I diritti fondamentali come conquiste sovrastatali di civiltà. II diritto di proprietà nelle CEDU, Torino, 2002, 39 ss., al quale rinvio per non ripetermi. In particolare, sul giudizio di ammissibilità ed il suo ruolo nella configurazione sostanziale di tale sistema di garanzia si v. 63 ss., anche solo per l'analisi della bibliografia citata.
}

REVISTA DO DIREITO UNISC, SANTA CRUZ DO SUL №. 43 | p.03-24 | MAIO-AGO 2014 
ricorrente, ma di una vera e propria riforma dei meccanismi statali di garanzia dei diritti, a prescindere dalle forme in cui questo possa avvenire ${ }^{12}$ : adeguamento della giurisprudenza e delle prassi interne ai livelli di garanzia imposti dal sistema CEDU; modifica legislativa; intervento della Corte costituzionale, ecc. Gli effetti delle singole sentenze della Corte europea non hanno, né possono avere infatti, portata normativa al pari di quelle della Corte di Giustizia. Ma il case-law della Corte può rivelare la necessità di un adeguamento sistematico del diritto interno al sistema CEDU ${ }^{13}$.

In passato la lettura dei rapporti tra ordinamento giuridico italiano e sistema CEDU effettuata dalla giurisprudenza statale aveva spesso ostinatamente insistito sulle differenze di prospettiva tra i due sistemi, a volte considerando gli obblighi derivanti dalla giurisprudenza di Strasburgo come circoscrivibili alle singole fattispecie concrete, diretta occasione delle relative pronunce. Soltanto con la più recente giurisprudenza della Corte costituzionale italiana - evidentemente mossa da una più ambiziosa pretesa di conformazione degli ordinamenti statali ai livelli europei di garanzia professata dalla giurisprudenza di Strasburgo - si è, finalmente, aperta la strada di una più intensa integrazione tra i sistemi di garanzia statale ed europeo dei diritti fondamentali ${ }^{14}$, in un'ottica di graduale ma sistematico ampliamento delle tutele in concreto offerte ai diritti ed alle libertà fondamentali. La già citata sentenza n. 317 del 2009 della Corte costituzionale, ad esempio, si è segnalata proprio per la più accentuata prospettiva di conformità allo spirito della Convenzione europea, non disvelando più alcuna forma di resistenza nei confronti della Corte di Strasburgo neanche in relazione alla tradizionale dottrina dei c.d. "controlimiti". II punto di fondo della più recente impostazione della Corte costituzionale è, infatti, finalmente rappresentato dalla specifica rilevanza attribuita alla "consistenza effettiva della tutela" dei diritti fondamentali nelle singole fattispecie, valutazione all'esito della quale verrà fatto

\footnotetext{
${ }^{12} \mathrm{Ne}$ ho dato ampliamente conto nel mio / diritti fondamentali, cit., $82 \mathrm{ss.}$

${ }^{13} \mathrm{Si}$ vedano le considerazioni formulate da M. Cartabia, Le sentenze "gemelle», cit., $3571 \mathrm{ss}$. Di recente sul tema si veda R. de Caria, Il bivio dopo Strasburgo: tutela effettiva o vittoria morale? L'obbligo per gli Stati di «conformarsi alle sentenze definitive della Corte» EDU nella prospettiva italiana, in Giur.cost., 2009, 2191 ss.

${ }^{14}$ Sui limiti del processo di integrazione nella stessa giurisprudenza costituzionale dell'epoca si veda, ad es., M. Ruotolo, La «funzione ermeneutica» delle convenzioni internazionali sui diritti umani nei confronti delle disposizioni costituzionali, in Dir.soc., 2000, 291 ss.
}

REVISTA DO DIREITO UNISC, SANTA CRUZ DO SUL №. 43 | p.03-24 | MAIO-AGO 2014 
obbligo al legislatore nazionale, al giudice comune o alla stessa Corte costituzionale di intervenire, secondo le rispettive competenze ed in relazione alla natura della violazione della Convenzione europea volta a volta denunciata, al fine di garantire che l'ordinamento italiano rimedi stabilmente "al deficit di tutela riguardo ad un diritto fondamentale". In ciò diviene rilevante, allora, il ruolo della stessa Corte costituzionale, laddove una sentenza di accoglimento fondata sul parametro di cui all'art. 117 Cost., integrato rispetto agli obblighi internazionali ex Convenzione europea, sia pronunciata proprio al fine di impedire che "continui ad avere efficacia nell'ordinamento giuridico italiano una norma di cui sia stato accertato il deficit di tutela riguardo ad un diritto fondamentale"15. Si tratta di ipotesi in cui appunto non viene in questione la semplice violazione una tantum di un diritto fondamentale, in un caso in cui la Corte europea sia intervenuta secondo la sua vocazione di giudice del caso singolo - per reagire al fallimento del sistema nazionale di protezione del diritto individuale, bensì dell'accertata illegittimità, ai sensi della CEDU, dei contenuti della disciplina normativa di fattispecie cui l'ordinamento italiano deve porre uno stabile rimedio per evitare il perpetuarsi della situazione di conflitto tra diritto interno e CEDU. II parametro di cui all'art. 117, primo comma Cost. aperto alla integrazione delle disposizioni CEDU quali norme interposte diventa, allora, un'efficace strumento idoneo allo scopo, concorrendo alla composizione di una equilibrata lettura degli impegni internazionali assunti dallo Stato all'atto della stipula della Convenzione europea e dei relativi protocolli addizionali. Nei casi di c.d. "violazione strutturale" della CEDU a rilevare non è infatti la forma del rimedio stabilmente assunto dall'ordinamento nazionale per conformarsi agli obblighi sanciti nella Convenzione, ma la effettiva efficacia sostanziale di tale rimedio, provenga esso da una norma di legge, da una prassi interpretativa delle giurisdizioni comuni o, appunto, da un intervento della Corte costituzionale ${ }^{16}$. Ma sul punto torneremo più avanti.

\footnotetext{
${ }^{15}$ Pto 7 del Considerato in diritto. Sulla questione si sofferma giustamente M. Cartabia, Le sentenze «gemelle», cit., 3571 ss.

${ }^{16}$ Op.ult.cit., 3571 ss.; F. Sorrentino, op.ult.cit., 219. Intervento della Corte costituzionale che tra l'altro non sempre è concretamente possibile, C. Pinelli, Sul trattamento giurisdizionale, cit., 3524 s.
} 


\section{II debito culturale della giurisprudenza di Strasburgo verso i sistemi}

\section{di Common Law in riferimento alla protezione dei diritti fondamentali}

Volendo riassumere gli elementi di più recente novità introdotti dalla giurisprudenza costituzionale italiana due sembrano i dati più rilevanti: in primo luogo una più accentuata apertura culturale al metodo comparativo ${ }^{17}$; e poi una più corretta specificazione del ruolo della categoria interpretativa dei c.d. "controlimiti" con esplicito riferimento al diritto CEDU, laddove assunto quale parametro nei giudizi di costituzionalità. Con riferimento al forte debito nei confronti della cultura giuridica anglosassone del metodo giurisprudenziale casistico proprio delle Corti europee avevo già avuto modo di soffermarmi ${ }^{18}$ sulla necessità di un serrato confronto con tale sistema in materia di protezione dei diritti fondamentali. Ciò in vista della forte dipendenza dei sistemi comunitario e CEDU di protezione dei diritti fondamentali dalla concezione anglosassone del principio di legalità, di fatto ricostruita nel Case-Law delle due Corti europee secondo i canoni classici del Common Law ${ }^{19}$. Albert Venn Dicey ${ }^{20}$ riteneva che esistessero due modelli di tutela dei diritti sotto il profilo della effettività delle garanzie: "(P)resso di noi i principi generali della costituzione (come per esempio il diritto alla libertà personale, o il diritto di riunione) sono il risultato di decisioni giurisprudenziali che hanno definito i diritti dei privati in particolari casi che sono

\footnotetext{
${ }^{17}$ In una diversa prospettiva si vedano gli interessanti sviluppi metodologici di tale approccio nel recente volume di G. Repetto, Argomenti comparativi e diritti fondamentali in Europa. Teorie dell'interpretazione e giurisprudenza sovranazionale, Napoli, 2011.

${ }^{18}$ I diritti fondamentali e la loro effettività, Relazione al Convegno sul tema Costituzione europea, costituzione economica, allargamento svoltosi presso l'Università di Teramo, Facoltà di Giurisprudenza, nei giorni 22-23 aprile 2005, in http://www.associazionedeicostituzionalisti.it/materiali/anticipazioni/diritti fondamentali bilancia/ nonché, in una versione integrata ed aggiornata, in Studi in memoria di Bruno Carboni, Napoli, 2010, 21 ss.

${ }^{19}$ A conferma delle tendenze evocate nel testo, con riferimento alla comparazione tra sistemi di Common Law e sistemi di Civil Law, si veda quanto osservato da A. Pizzorusso, Le sentenze dei giudici costituzionali tra diritto giurisprudenziale $e$ diritto legislativo, in http://www.costituzionalismo.it/articolo.asp?id=247, 2/2007 in relazione al ruolo acquisito oggi dalle giurisdizioni costituzionali nei paesi a diritto legislativo. L'autore parla, infatti, di "ambiente nel quale la tradizionale contrapposizione di civil law e common law" ha "cominciato a perdere quel ruolo di summa divisio ai fini dell'indagine comparatistica che gli era stato assegnato in passato"

${ }_{20}$ Introduzione allo studio del diritto costituzionale. Le basi del costituzionalismo inglese (1885), 1915, tr.it della versione inglese del 1959, rist. London-New York, 1964, a cura di A. Torre, Bologna, 2003, $162 \mathrm{~s}$. che, come ricorda oggi P. Leyland, Introduzione al diritto costituzionale del Regno Unito, Torino, 2005, 7, "propone la spiegazione forse più esauriente delle nozioni-cardine di sovranità parlamentare e di principio della Rule of Law".
}

REVISTA DO DIREITO UNISC, SANTA CRUZ DO SUL №. 43 | p.03-24 | MAIO-AGO 2014 
stati portati alla cognizione delle corti di giustizia, laddove invece in molte forme costituzionali straniere la protezione (...) che è data ai diritti individuali risulta, 0 sembra risultare, dai principi generali della costituzione". Dal confronto tra i due sistemi di garanzia dei diritti, quello europeo continentale fondato sulle proclamazioni formali proprie delle Carte dei diritti e quello inglese, basati sulla strutturale coincidenza tra gli strumenti di garanzia del diritto individuale e la disciplina del diritto individuale stesso ${ }^{21}$ Dicey ricostruiva il contenuto essenziale della tutela costituzionale dei diritti propria dell'ordinamento anglosassone nella stessa effettività degli strumenti di garanzia, nella concretezza dei rimedi per la loro protezione.

A ben vedere il sistema di tutela dei diritti fondamentali proprio delle Corti europee e, per quanto qui di interesse, professato nella giurisprudenza della Corte di Strasburgo, si caratterizza per il fatto che la disciplina dei diritti fondamentali sia individuata negli stessi strumenti di garanzia, "deducendo quella da questi", al fine di costruire una vera e propria "dottrina dei rimedi, come dire, delle effettive garanzie offerte dall'ordinamento a protezione dei diritti dei singoli”22. E se leggiamo con attenzione gli esiti della citata sentenza n. 317 della Corte costituzionale italiana, come pure delle successive pronunce riportate più avanti, l'approccio culturale che emerge sembra proprio rispondere a tale paradigma culturale, orientato a cogliere gli elementi in concreto della effettiva tutela offerta al singolo diritto nella comparazione tra diritto interno e diritto CEDU. L'obiettivo esplicitamente professato, infatti, è quello di assicurare un "ampliamento di tale tutela" piuttosto che non, all'opposto, una sua sostanziale diminuzione, incentrando il proprio giudizio sulla integrazione del parametro di costituzionalità mediante il rinvio alle disposizioni della CEDU ed alla giurisprudenza di Strasburgo nei termini di un confronto tra "deficit di garanzie" ed "espansione delle garanzie", affinché "il risultato complessivo dell'integrazione delle garanzie dell'ordinamento" sia sempre "di segno positivo, nel senso che

\footnotetext{
${ }^{21}$ "Ubi jus ibi remedium", A.V. Dicey, op.ult.cit., 165 s., e 171 ss. con riferimento ai singoli diritti di libertà.

${ }_{22}^{22}$. Bilancia, I diritti fondamentali e la loro effettività, cit., § 3 , in fine.
}

REVISTA DO DIREITO UNISC, SANTA CRUZ DO SUL №. 43 | p.03-24 | MAIO-AGO 2014 
dall'incidenza della singola norma CEDU sulla legislazione italiana deve derivare un plus di tutela per tutto il sistema dei diritti fondamentali"23.

E se l'obiettivo deve essere la valutazione della effettività consistenza in concreto degli strumenti di garanzia dei diritti fondamentali diviene, allora, assai rilevante il confronto tra la disciplina di tali rimedi per come ricomposta nel sistema della giurisprudenza della Corte di Strasburgo, da una parte, e la disciplina costituzionale e legislativa dei diritti fondamentali, dall'altra. Ed è qui che la Corte costituzionale sembra aggiungere un ulteriore rilevante elemento alla lettura dei rapporti tra Costituzione e CEDU per come ricostruita a partire dalle sentenze del 2007. Afferma la Corte che "nel concetto di massima espansione delle tutele deve essere compreso, come già chiarito nelle sentenze nn. 348 e 349 del 2007, il necessario bilanciamento con altri interessi costituzionalmente protetti, cioè con altre norme costituzionali". Ma norme, aggiunge poi la Corte, "che a loro volta garantiscano diritti fondamentali"24. Tra le norme costituzionali con cui bilanciare le disposizioni CEDU, pertanto, la Corte costituzionale sembra dare rilievo qui soprattutto alle disposizioni sugli stessi diritti costituzionali. II che rappresenta un'interessante novità ed un'importante presa di posizione, salvo quanto verremo osservando più avanti. Come è noto, infatti, il sistema CEDU riconosce agli Stati membri un ampio "margine di apprezzamento" nella composizione in equilibrio della tutela del singolo diritto con gli interessi generali che ne possano giustificare in concreto una compressione ${ }^{25}$, pur sempre compatibile con la Convenzione medesima se si dimostri che possa essere ritenuta una limitazione "necessaria in una società democratica". E le istituzioni nazionali sono spesso state considerate, dalla giurisprudenza di Strasburgo, più idonee a valutarne la consistenza effettiva rispetto alla stessa Corte EDU. La dottrina del "margine di apprezzamento"26, cioè, ha spesso assunto, nella giurisprudenza CEDU, il valore di clausola generale a fattispecie indeterminata in

\footnotetext{
${ }^{23}$ Pto 7 del considerato in diritto.

${ }^{24}$ Loc.ult.cit.

${ }^{25}$ Si vedano, infatti, gli interrogativi posti da A. Guazzarotti, La Corte e la CEDU, cit., 3579, a commento della sent. n. 349 del 2007.

${ }^{26}$ Rinvio alla bibliografia citata nel mio I diritti fondamentali come conquiste sovrastatali, cit., spec. 113 ss. Si veda, da ultimo, il saggio di S. Mancini, La supervisione europea presa sul serio: la controversia sul crocifisso tra margine di apprezzamento e ruolo contro-maggioritario delle Corti, in Giur.cost., 2009, 4055 ss.
} 
grado di affievolire il rigore del sindacato sulle violazioni dei diritti fondamentali nei singoli casi concreti. E la giurisprudenza costituzionale qui richiamata fa costante riferimento a tale dottrina, quale "temperamento alla rigidità dei principi formulati in sede europea". Temperamento affidato in primo luogo alla funzione legislativa del Parlamento ma altresì esercitabile, ove necessario anche a causa della assenza di un equilibrato intervento legislativo, dalla stessa Corte costituzionale.

Il punto è che ora la Corte costituzionale italiana dichiara di voler individuare gli "altri" interessi costituzionali con cui bilanciare, in un'ottica di funzionalizzazione dell'interesse generale, la disciplina costituzionale dei diritti fondamentali nei diritti fondamentali medesimi ${ }^{27}$, con riferimento primario a quelli che potrebbero trovarsi in concreto conflitto reciproco. Così, appunto, la Corte costituzionale nella citata sentenza n. 317: "In sintesi, il «margine di apprezzamento» nazionale può essere determinato avuto riguardo soprattutto al complesso dei diritti fondamentali, la cui visione ravvicinata e integrata può essere opera del legislatore, del giudice delle leggi e del giudice comune, ciascuno nell'ambito delle proprie competenze"28. Nella lettura della Corte, quindi, la disciplina dei singoli diritti fondamentali sembrerebbe poter subire una ponderazione essenzialmente in funzione della garanzia di altri diritti fondamentali, escludendo in linea di principio la rilevanza di più generici "interessi

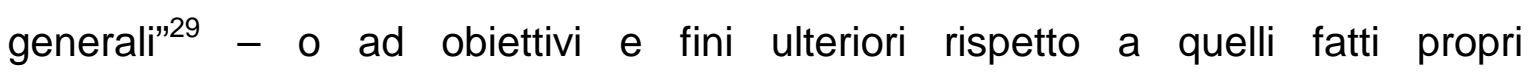
dall'ordinamento costituzionale medesimo. E' come se la Corte costituzionale ritenesse di dover rinunciare al margine di apprezzamento che pure la giurisprudenza CEDU riconosce agli Stati membri ${ }^{30}$ a tutela dei loro superiori interessi, rinvenendo in ultima istanza negli stessi diritti costituzionali tali supremi interessi. Ritrovandosi così un'ulteriore giustificazione alla sostanziale differenza tra sistema CEDU e diritto comunitario e dell'UE, come noto portatore di una

\footnotetext{
${ }^{27}$ Si veda quanto osserva R. Niro, Il vento di Strasburgo sui diritti inviolabili, cit., §§ 3 e 5 ..

28 Pto. 7 del considerato in diritto, ultimo cpv. Sul punto già O. Pollicino, Margine di apprezzamento, art. 10, c.1, Cost. e bilanciamento "bidirezionale": evoluzione o svolta nei rapporti tra diritto interno e diritto convenzionale nelle due decisioni nn. 311 e 317 del 2009 della Corte costituzionale?, in Forum dei Quad.cost., 16 dicembre 2009.

${ }^{29}$ Si vedano, infatti, le illuminanti considerazioni di A. Pace, La limitata incidenza della C.E.D.U. sulle libertà politiche e civili in Italia, in Dir.pubbl., 2001, spec. 11 ss.

${ }_{30} \mathrm{Si}$ veda F. Angelini, Ordine pubblico e integrazione costituzionale europea. I principi fondamentali nelle relazioni interordinamentali, Padova, 2007, spec. 129 ss., 226 ss.
} 
dottrina funzionalista anche in relazione alla disciplina dei diritti fondamentali ${ }^{31}$. Ma questa apparente conclusione di sintesi andrà parzialmente corretta alla luce dei più recenti interventi giurisprudenziali, pur rimanendo confermata nei propri elementi essenziali di fondo, soprattutto in ordine alla tendenza ad una sostanziale costituzionalizzazione dell'interesse generale in ogni operazione di bilanciamento che fosse ritenuta necessaria all'atto del riconoscimento del concreto livello di garanzia, nel sistema costituzionale italiano, di uno dei diritti e delle libertà fondamentali garantiti dalla Convenzione europea.

\section{II caso delle leggi retroattive ed il problema dell'incidenza del potere} legislativo sull'esercizio della funzione giurisdizionale

Già nella sentenza n. 311 del 2009 la Corte costituzionale italiana aveva preso posizione con riferimento agli effetti delle garanzie dei diritti alla tutela giurisdizionale di cui all'art. 6 CEDU nei casi di interventi legislativi ad efficacia retroattiva atti ad incidere sull'esercizio della funzione giurisdizionale nei processi in corso al momento dell'entrata in vigore delle nuove norme. Nella sentenza richiamata $^{32}$, ancorché di rigetto, la Corte costituzionale aveva infatti affermato: "il principio dello Stato di diritto e la nozione di processo equo sancito dall'art. 6 della CEDU vietano l'interferenza del legislatore nell'amministrazione della giustizia destinata a influenzare l'esito della controversia fatta eccezione che per motivi imperativi di interesse generale ${ }^{33 "}$ con ciò richiamando la copiosa giurisprudenza CEDU che "censura la prassi di interventi legislativi sopravvenuti, che modifichino retroattivamente in senso sfavorevole per gli interessati le disposizioni di legge attributive di diritti, la cui lesione abbia dato luogo ad azioni giudiziarie ancora pendenti all'epoca della modifica".

La questione dell'incidenza delle leggi retroattive sull'esercizio in concreto del diritto alla tutela giurisdizionale diviene, però, l'occasione per richiamare

\footnotetext{
${ }^{31}$ Il tema è, come noto, estremamente complesso. Si vedano, ad esempio, le riflessioni di cui ai saggi di G. Azzariti ora raccolti in Id., Studi sui diritti in Europa, Roma, 2006.

${ }_{32}$ Pto 8 del considerato in diritto.

${ }^{33}$ Dalla Corte individuati in fattispecie del tipo: "ragioni storiche epocali, come nel caso della riunificazione tedesca"; o la "sussistenza di una «imperfezione» tecnica, nel contesto normativo originario".
} 
l'attenzione, da parte della Corte costituzionale, sulle differenze che caratterizzano i due sistemi di garanzia dei diritti fondamentali, con particolare riferimento al ruolo delle due Corti. La diversa posizione di esse nei rispettivi ordinamenti riduce considerevolmente l'utilità di un acritico confronto diretto tra le fattispecie oggetto dei relativi giudizi, quand'anche esse dovessero apparire a prima vista analoghe. La Corte EDU e la Corte costituzionale italiana hanno fondamento giuridico-costituzionale, collocazione istituzionale, attribuzioni differenti che si riflettono, pur nel contesto di un'incessante interazione reciproca, sugli esiti degli interventi giurisprudenziali di ciascuna e sugli effetti giuridici delle relative pronunce ${ }^{34}$. Nella recentissima sentenza n. 264 del 2012, ad esempio, questa impostazione rappresenta un importante elemento di qualificazione del "dialogo" tra le due Corti proprio con riferimento alle linee di continuità e, all'opposto, ai distinguo rispetto ai precedenti giurisprudenziali ${ }^{35}$. Gli interventi delle due Corti sulle medesime questioni, infatti, non possono affatto essere considerati del tutto sovrapponibili ${ }^{36}$. Così accade, ad esempio, confrontando le soluzioni rinvenibili nella giurisprudenza delle due Corti con riferimento alla appena richiamata questione del se siano ammissibili o meno leggi retroattive che, incidendo su processi in corso, ne condizionino gli esiti compromettendo l'effettivo esercizio del diritto alla tutela giurisdizionale di una delle parti in causa. Su tale questione torneremo al termine di questo scritto, perché in effetti rappresenta un interessante terreno di confronto tra la giurisprudenza CEDU e quella della nostra Corte costituzionale, vista la reale distanza tra le rispettive posizioni. Ma per il momento il quesito ci è utile per confrontare in concreto le differenti attribuzioni e ruoli delle due Corti insieme agli effetti delle relative pronunce nel sistema costituzionale.

\footnotetext{
${ }^{34}$ Secondo l'impostazione di metodo esemplarmente seguita, ad esempio, da A. Cerri, Corso di Giustizia costituzionale plurale, Milano, 2012, 541 ss. Con riferimento alla CEDU si veda, nello stesso senso, F. Gallo, Rapporti tra Corte costituzionale e Corte EDU, in AIC, Rivista, 1/2012, 1. Si tratta dell'approccio critico di recente adottato anche nell'interessante studio di D. Tega, I diritti in crisi. Tra Corti nazionali e Corte europea di Strasburgo, Milano, 2012, spec. $41 \mathrm{ss}$., 51 ss., spec. 88 ss., 101 ss., 153 ss.

${ }^{35} \mathrm{Ci}$ si riferisce alla giurisprudenza aperta con le due pronunce del 2007, nn. 348 e 349 citate

${ }^{36} \mathrm{Si}$ vedano gli interessanti sviluppi della riflessione di O. Pollicino, $A$ further argument in favour of the construction of a general theory of the domestic impact of jurisprudential supranational law. The genesis and the first steps of ECHR and EU legal orders, in Comparative Law Review, 3/2012, 1 ss., spec. 10 ss., 20 ss.
}

REVISTA DO DIREITO UNISC, SANTA CRUZ DO SUL №. 43 | p.03-24 | MAIO-AGO 2014 


\section{Segue. I limiti al divieto di retroattività delle leggi nel sistema della Corte europea dei diritti dell'Uomo}

Per costante giurisprudenza della Corte europea dei diritti dell'uomo "il principio della preminenza del diritto e la nozione di equo processo di cui all'art. 6 della Convenzione si oppongono, salvo che per imperative ragioni di interesse generale $^{37}$, all'ingerenza del potere legislativo nell'amministrazione della giustizia con lo scopo di influire sull'esito giudiziario della controversia" riducendo di fatto significativamente la facoltà dello Stato di intervenire su una vicenda oggetto di un giudizio con una nuova disciplina legislativa ad effetto retroattivo ${ }^{38}$. E la Corte costituzionale ha, in più di un'occasione, di recente avuto modo di fare proprio tale principio di diritto, addirittura assumendone il tenore letterale nella motivazione delle proprie stesse sentenze ${ }^{39}$. Come vedremo, però, in sede di applicazione alle fattispecie concrete di tale principio, sui "motivi imperativi di interesse generale" che soli giustificherebbero eccezioni alla regola la Corte costituzionale italiana si è mostrata molto più indulgente, generando un apparente contrasto giurisprudenziale, se non fosse appunto per il differente contesto in cui vanno ad inserirsi le pronunce delle due diverse Corti.

Nelle precedente sentenza n. 317 del 2009 la Corte costituzionale italiana aveva esplicitamente affermato che il riconoscimento dei diritti e delle libertà garantiti dalla Convenzione europea dei diritti dell'uomo, nel processo di

\footnotetext{
${ }^{37}$ Corsivo mio.

${ }^{38}$ La giurisprudenza CEDU è, sul punto, ormai consolidata. Ex plurimis si vv. CEDU, sez. 2, 14 febbraio 2012, Arras et al. c. Italia, (17972/07) pti 42 ss. della motivazione in diritto; Id., sez. 2, 7 giugno 2011, Agrati et al. c. Italia (ricc. nn. 43549/08, 6107/09, 5087/09) pti 58 ss., della motivazione in diritto; Id., sez. 2, 31 maggio 2011, Maggio et al. c. Italia (46286/09), pti 43 ss. della motivazione in diritto, pto 3.1.3 della motivazione in diritto; Id., sez.5, 25 novembre 2010, Lilly France c. France, (20429/07) pti 46 ss. della motivazione in diritto; Id., sez. 5, 11 febbraio 2010, Javaugue c. France (39730/06), pti 37 ss. della motivazione in diritto; Id., Grande Camera, 10 giugno 2008, Bortesi et al. c. Italia, (71399/01) pti 40 ss. della motivazione in diritto; Id., Grande Camera, 29 marzo 2006, Scordino c. Italia (36813/97) pti 127 ss. della motivazione in diritto. Si v., ancora, D. Tega, I diritti in crisi, cit., 173 ss.

${ }^{39}$ Sentenze nn. 311 del 2009, pti 8 ss. del Considerato in diritto, pur di rigetto, con commento di M. Massa, La "sostanza» della giurisprudenza europea sulle leggi retroattive, in Giur.cost., 2009, 4679 ss, e 78 del 2012, pto 13 del Considerato in diritto, in Giur.cost., 2012, 1017 ss., con commento rigorosamente argomentato in tema di leggi c.d. di interpretazione autentica di G.U. Rescigno, Leggi di interpretazione autentica, leggi retroattive e possibili ragioni della loro incostituzionalità, ivi, 1072 ss.
}

REVISTA DO DIREITO UNISC, SANTA CRUZ DO SUL №. 43 | p.03-24 | MAIO-AGO 2014 
assorbimento dei relativi strumenti di garanzia all'interno del sistema italiano di protezione dei diritti costituzionali deve condurre necessariamente "alla massima espansione delle garanzie" e che in tale massima espansione debba essere "compreso il necessario bilanciamento con altri interessi costituzionalmente protetti, cioè con altre norme costituzionali, che a loro volta garantiscano diritti fondamentali che potrebbero essere incisi dall'espansione di una singola tutela". In sintesi riconducendo, come osservato più sopra, il «margine di apprezzamento» statale al complesso dei diritti fondamentali" ${ }^{\prime 40}$. Così ancora nella successiva importante pronuncia n. 113 del 2011 sono proprio "le garanzie attinenti a diritti fondamentali della persona", "pur nella indubbia rilevanza dei valori della certezza e della stabilità della cosa giudicata", ad ispirare l'accoglimento della questione di legittimità costituzionale, addirittura prevedendo un nuovo caso di revisione della sentenza o del decreto penale di condanna, ai sensi dell'art. 630 c.p.p., al fine di consentire la riapertura di un processo per conformarsi ad una sentenza definitiva di condanna pronunciata dalla Corte europea nei confronti dell'Italia ${ }^{41}$.

Questo approccio fortemente espansivo del valore delle garanzie costituzionali dei diritti riceve formale ossequio anche nella citata sentenza n. 264 del $2012^{42}$, sebbene la applicazione in concreto del principio del "necessario bilanciamento con altri interessi costituzionalmente protetti" produca qui un risultato di apparente contrazione dei livelli di tutela assicurati al singolo. Almeno, all'esito della pronuncia di rigetto, con riferimento alla posizione del ricorrente e a dispetto di una pronuncia favorevole della Corte europea dei diritti dell'uomo sulla

\footnotetext{
${ }^{40}$ Pto 7 del Considerato in diritto. Si v., in proposito, anche L. Cassetti, La «ricerca dell'effettività»: dalla lotta per l'attuazione dei principi costituzionali all'obiettivo della "massima espansione delle tutele», in Id. (a cura di), Diritti, principi e garanzie sotto la lente dei giudici di Strasburgo, Napoli, 2012, 3 ss.

${ }^{41}$ La sentenza si segnala per lo specifico ruolo assunto dalla Corte costituzionale quale strumento di esecuzione di una precedente sentenza CEDU ai sensi dell'art. 46 della Convenzione stessa. Sul punto rinvio senz'altro ai commenti di G. Ubertis, La revisione successiva a condanne della Corte di Strasburgo; G. Repetto, Corte costituzionale e CEDU al tempo dei conflitti sistemici; S. Lonati, La Corte costituzionale individua lo strumento per adempiere all'obbligo di conformarsi alle condanne europee: l'inserimento delle sentenze della Corte europea tra $i$ casi di revisione, in Giur.cost., 2011, risp. 1542 ss., 1548 ss., 1557 ss. ed alle più ampie riflessioni di A. Cardone, voce Diritti fondamentali (tutela multilivello dei), in Enc.dir. Annali, IV, Milano, 2011, spec. 393 ss. e di R. Caponi, Corti europee e giudicati nazionali, http://www.astrid-online.it/--giustizi/Studi-ric/Caponi Rel-Congresso-Ass-processo-civile 25-26 09 09.pdf
}

${ }^{42} \mathrm{Si} \mathrm{v}$. i pti 4.1 e 5.1 del Considerato in diritto.

REVISTA DO DIREITO UNISC, SANTA CRUZ DO SUL №. 43 | p.03-24 | MAIO-AGO 2014 
medesima fattispecie ${ }^{43}$. E l'argomento utilizzato dalla Corte per ammorbidire l'esito della funzionalizzazione del margine di apprezzamento statale alla più accentuata tutela degli stessi diritti fondamentali e ricondurlo al tradizionale ruolo di clausola di "temperamento alla rigidità dei principi formulati in sede europea" è dato da una forte accentuazione del valore del criterio della "valutazione sistemica e non isolata dei valori coinvolti ${ }^{34}$. II che conduce, ovviamente, alla necessità di un "bilanciamento, solo ad essa spettante" 45 degli interessi in conflitto in considerazione dei propri ruolo e posizione nel sistema costituzionale di garanzia dei diritti, in quanto "la tutela dei diritti fondamentali deve essere sistemica e non frazionata in una serie di norme non coordinate ed in potenziale conflitto tra loro". E l'esito del giudizio si fonderà così su questo assunto e sulla conseguente prevalenza "degli interessi antagonisti" a quello del ricorrente, rispetto ad esso "di pari rango costituzionale" ma che conducono, a giudizio della Corte italiana e nel senso che essa attribuisce a tale nozione, all'accertamento della sussistenza, nel caso di specie, di "quei preminenti interessi generali che giustificano il ricorso alla legislazione retroattiva" ammessa, quale eccezione al principio, dalla stessa Corte europea. Sul punto torneremo in chiusura. Ma è bene ricordare che in un'analoga vicenda, con riferimento al medesimo provvedimento legislativo ad efficacia retroattiva oggetto del giudizio di cui alla sentenza n. 264, la Corte europea era giunta ad una conclusione, opposta, di condanna dell'Italia ${ }^{46}$ con una sentenza non a caso qui invocata dal giudice a quo ad integrazione del parametro di giudizio indicato nell'art. 117, comma 1 Cost. in relazione all'art. 6, paragrafo 1 della CEDU.

A parziale apparente correzione dei propri precedenti pronunciamenti la Corte costituzionale italiana, quindi, assorbe le garanzie dei diritti fondamentali imposte dal diritto CEDU in un bilanciamento articolato non più soltanto tra

\footnotetext{
${ }^{43}$ Si tratta della sentenza del 31 maggio 2011, Maggio et al. c. Italia (46286/09), cit., riportata negli atti di causa e richiamata supra nt. 38).

${ }^{44} \mathrm{G}$. Repetto, Corte costituzionale e CEDU al tempo dei conflitti sistemici, cit., loc.cit.

${ }^{45}$ Pto 5.4 del Considerato in diritto, corsivo mio.

${ }^{46}$ Si tratta della citata sentenza sul caso Maggio et al. c. Italia, cit., stabilendo una condanna dell'Italia proprio per violazione del diritto ad un equo processo dei ricorrenti a causa dell'intervento della I. n. 296 del 2006, art. 1 comma 777 - oggetto dello stesso giudizio di cui alla citata sentenza n. 264 della Corte costituzionale italiana - per essere lo Stato italiano intervenuto nel corso di un giudizio con disposizioni normative ad efficacia retroattiva "per garantire che l'esito del procedimento in cui esso era parte gli fosse favorevole".
}

REVISTA DO DIREITO UNISC, SANTA CRUZ DO SUL №. 43 | p.03-24 | MAIO-AGO 2014 
elementi omogenei, i diritti fondamentali stessi alla ricerca della massima espansione complessiva delle tutele, ma in un'ottica di maggiore complessità come nel caso da ultimo richiamato - con più amplio riferimento ad altri "principi, diritti e beni di rilievo costituzionale". Elementi normativi questi che, con riferimento ai limiti della retroattività di leggi che rischiano di risolversi in un'illegittima "interferenza del corpo legislativo nell'amministrazione della giustizia con il proposito di influenzare la determinazione giudiziaria di una controversia", violando quindi l'art. 6 della CEDU, consentono ora alla Corte costituzionale di individuare adeguati "motivi imperativi di interesse generale" che, ai sensi della giurisprudenza della Corte europea ma in un caso in cui la stessa Corte europea ne escludeva la sussistenza, ne giustificano la conformità a Costituzione. Assumendo il parametro costituzionale proprio per come integrato, sul piano delle garanzie dei diritti, dalla stessa Convenzione europea e dalla giurisprudenza della Corte di Strasburgo. Rispetto alle precedenti pronunce della Corte costituzionale questo essenziale passaggio rappresenta, allora, un apparente ripensamento ${ }^{47}$

\section{Prime conclusioni: il differente ruolo delle due Corti nel sistema costituzionale europeo}

In realtà la soluzione prospettata è del tutto coerente con il sistema dei rapporti tra Costituzione e CEDU, tra Corte costituzionale e Corte di Strasburgo per come venutosi a delineare a far data dalle citate sentenze del 2007. II suo inquadramento nel contesto dei rapporti ordinamentali tra le due Corti è agevole se solo si assumano adeguate prospettive di analisi, riflettendo sulle distinte attribuzioni e sul diverso ruolo delle due Corti; sugli effetti delle relative sentenze, non assimilabili con riferimento alla loro portata normativa ed alla platea dei potenziali destinatari; agli obblighi degli Stati membri di conformarsi alla giurisprudenza della Corte europea che variano notevolmente a seconda che si tratti di una violazione occasionale, in un caso singolo, di un diritto o di una libertà garantite dalla CEDU o, all'opposto, di una violazione sistematica, come si suol

${ }^{47}$ Si veda già, peraltro, S. Foà, Un conflitto di interpretazione tra Corte costituzionale e Corte europea dei diritti dell'uomo: leggi di interpretazione autentica e ragioni imperative di interesse generale, in www.federalismi.it, 15/2011.

REVISTA DO DIREITO UNISC, SANTA CRUZ DO SUL №. 43 | p.03-24 | MAIO-AGO 2014 
dire, strutturale della Convenzione. Partendo da tale ultima questione è ormai a tutti noto, infatti, che gli obblighi di adattamento gravanti sugli ordinamenti interni sono del tutto diversi a seconda che la condanna dello Stato dipenda da un cedimento in un caso concreto, ed isolato, del sistema delle garanzie dei diritti in seno ad un ordinamento statale comunque idoneo, di norma, ad offrire un livello adeguato di protezione dei diritti fondamentali equivalente, se non superiore, a quello imposto dal sistema CEDU; o, viceversa, sia il frutto di una sistematica violazione della Convenzione a causa di un difetto, appunto, strutturale, del sistema statale di garanzie che in virtù di una legge, o di una prassi amministrativa o di una giurisprudenza di merito consolidate generi una stabile, costante incompatibilità dell'ordinamento statale con le garanzie offerte dal sistema CEDU. Violazione sistematica che, ben oltre l'intervento riparatore nel singolo caso concreto, imporrà allo Stato membro di intervenire sulle cause di tale inadeguatezza, con una riforma legislativa o amministrativa, un solido mutamento giurisprudenziale, l'impegno di particolari risorse economiche, ecc. Un conto è, insomma, riparare ad un singolo errore giudiziario in un sistema comunque idoneo in astratto ad offrire un livello di tutela adeguato agli standard imposti dalla CEDU; un conto è dotarsi di nuovi e più efficaci strumenti di protezione dei diritti nei casi di conclamata inidoneità dell'ordinamento statale a garantire tale standard a causa di una evidente insufficienza ordinamentale ${ }^{48}$.

La soluzione di un caso concreto, al di là della forza di precedente che una decisione adottata in un sistema ispirato dai principi del Common Law - il sistema CEDU - possa assumere in un sistema consolidato di Civil Law - l'ordinamento italiano - è cosa diversa dal mutamento della disciplina legislativa o della prassi giurisprudenziale nazionali ${ }^{49}$. Le sentenze della Corte europea non hanno, non

\footnotetext{
${ }^{48}$ La questione è, come accennato, molto nota e presente da tempo nel dibattito scientifico più avvertito. Si veda già il mio I diritti fondamentali come conquiste sovrastatali di civiltà, cit.; M. Cartabia, La CEDU e l'ordinamento italiano: rapporti tra fonti, rapporti tra giurisdizioni, in AA.VV., All'incrocio tra Costituzione e CEDU. Il rango delle norme della Convenzione e l'efficacia interna delle sentenze di Strasburgo, Torino, 2007, 1 ss., spec. 17 ss.; Id., La Convenzione europea dei diritti dell'uomo e l'ordinamento italiano, in A. Balsamo, R.E. Kostoris (a cura di), Giurisprudenza europea e processo penale italiano, Torino, 2008, 33 ss., spec. 56 ss., 62 ss.; A. Cardone, voce Diritti fondamentali, cit., 389 ss.; P. Pirrone, Art. 46, in S. Bartole, P. De Sena, V. Zagrebelsky (a cura di), Commentario breve alla Convenzione europea dei diritti dell'uomo, Padova, 2012, 744 ss., spec.

${ }^{49}$ Sul punto la riflessione di D. Tega, I diritti, cit., spec. 43 ss., 154 ss.
}

REVISTA DO DIREITO UNISC, SANTA CRUZ DO SUL №. 43 | p.03-24 | MAIO-AGO 2014 
possono avere, effetti propriamente normativi nell'ordinamento italiano. Non ne modificano il diritto oggettivo con interventi sistematici. Mentre le sentenze di accoglimento della Corte costituzionale, riproducano o meno nell'ordinamento interno gli effetti positivi del riconoscimento di un diritto o di una libertà fondamentali secondo la soluzione interpretativa adottata dalla Corte di Strasburgo nel singolo caso concreto, dispiegano i loro effetti sul tessuto normativo innovando, con efficacia erga omnes, al diritto oggettivo modificandone stabilmente i contenuti. Elementi differenziali, questi, di cui sarebbe insensato non tenere conto nel confrontare gli esiti della giurisprudenza sui diritti fondamentali nei due differenti contesti. Una pronuncia di accoglimento della Corte costituzionale non può, pertanto, essere invocato nei casi di violazione occasionale della stessa Convenzione al solo fine di dare seguito ad una sentenza di condanna della Corte europea, perché intervenendo sul sistema normativo nel suo complesso questa dovrà necessariamente tenere conto di tutti $\mathrm{i}$ potenziali effetti di un intervento caducatorio con efficacia erga omnes ${ }^{50}$. Non sempre, cioè, il principio di diritto enunciato nella sentenza CEDU può essere trattato nell'ordinamento interno - dal giudice comune e, ancor di più, dalla Corte costituzionale - come se fosse una "«norma di legge" dimenticando che la pronuncia della Corte è legata a doppio filo ad un caso concreto" "51. L'espansione degli effetti della giurisprudenza europea nell'ordinamento italiano diviene, invece, necessario nei casi di violazione reiterata della CEDU, sintomo di un problema di sistema, di una carenza oggettiva, strutturale, del diritto italiano rispetto agli obblighi assunti con la Convenzione. In tal caso, ovviamente, oltre ad un intervento del legislatore ben si potrebbe invocare una sentenza di accoglimento della Corte costituzionale.

In questa prospettiva andrebbero, quindi, inquadrate le questioni connesse, con riferimento alla problematica degli strumenti idonei a dare esecuzione agli obblighi scaturenti dalle sentenze di condanna della Corte europea, con l'adozione, da parte della stessa Corte, di sentenze c.d. pilota, proprio al fine di

${ }^{50}$ F. Gallo, Rapporti tra Corte costituzionale e Corte EDU, cit., 2. A. Cardone, voce Diritti fondamentali, cit., $408 \mathrm{~s}$. fa riferimento ad una vera e propria "funzione nomofilattica" della giurisprudenza della Corte costituzionale.

${ }^{51}$ D. Tega, op.cit., 44, 101 ss., 156 ss. Assai interessanti, in una diversa prospettiva, le riflessioni di G. Repetto, Argomenti comparativi e diritti fondamentali in Europa, cit., spec. 305 ss.

REVISTA DO DIREITO UNISC, SANTA CRUZ DO SUL №. 43 | p.03-24 | MAIO-AGO 2014 
indurre a più stabili azioni di adattamento dell'ordinamento statale al sistema CEDU. Ma in questa sede non sarà possibile andare oltre il rinvio alle più recenti riflessioni sull'art. 46 della Convenzione europea e alla riforma dei meccanismi procedurali di esecuzione delle sentenze avutasi con l'entrata in vigore, nell'anno 2010, del Protocollo n. 14 alla Convenzione ${ }^{52}$.

Su tali premesse è, forse, allora possibile ricondurre l'esito della recente sentenza n. 264 del 2012 ad una posizione di rinnovata coerenza con il sistema CEDU malgrado l'apparente contrasto delle relative posizioni sostanziali. La pronuncia della Corte italiana, infatti, non illumina soltanto il caso concreto, il giudizio a quo in sé e per sé perché affronta un giudizio su norme. Perché l'efficacia di un dispositivo di accoglimento avrebbe effetto sul tessuto normativo generale, Avrebbe, appunto, effetti sistemici sull'ordinamento complessivamente inteso. Di qui l'esito del bilanciamento dei contenuti delle prestazioni patrimoniali invocate dal ricorrente ed incise, nel loro computo, dalle nuove disposizioni ad efficacia retroattiva, risolto in base ad una ponderazione riferita al sistema previdenziale nel suo complesso, alla corrispondenza tra risorse disponibili e prestazioni erogate a garanzia della "razionalità complessiva del sistema stesso (...) impedendo alterazioni della disponibilità economica a svantaggio di alcuni contribuenti ed a vantaggio di altri, e così garantendo il rispetto dei principi di eguaglianza e di solidarietà” ${ }^{\prime 3}$. Tutti interessi di rango costituzionale "antagonisti" della pretesa in concreto dei ricorrenti ma oggettivamente bilanciati nella disciplina legislativa dei diritti fondamentali evocati con gli equivalenti diritti previdenziali degli altri contribuenti. L'eguaglianza sostanziale nel regime delle prestazioni apparendo, nel caso concreto, più rilevante del rispetto dell'equilibrio di bilancio ai sensi dell'art. 81 Cost. II confronto diretto tra i contenuti delle

\footnotetext{
${ }^{52}$ Firmato a Strasburgo il 13 maggio 2004. Sul punto, diffusamente, P. Pirrone, Art. 46, in S. Bartole, P. De Sena, V. Zagrebelsky (a cura di), Commentario breve alla Convenzione europea, cit., 744 ss., 747 ss., 756 ss., 760 ss.; S. Vezzani, L'attuazione delle sentenze della Corte europea dei diritti dell'uomo che richiedono l'adozione di misure a portata generale, in L. Cassetti (a cura di), Diritti, principi e garanzie, cit., 43 ss., 54 ss., 62 ss.; F. Gallo, Rapporti tra Corte costituzionale e Corte EDU, cit., 3. II rilievo di tale disposizione è stato oggetto di specifica attenzione nella citata sentenza n. 113 del 2011 della Corte costituzionale, pti 4 ss. del Considerato in diritto. Di tale prospettiva di riforma, da tempo presente nel dibattito, avevo già dato conto nel mio I diritti fondamentali come conquiste, cit., 91. Si vedano, infine, le interessanti considerazioni di V. Zagrebelsky, Note sulle conclusioni della Conferenza di Brighton "per assicurare l'avvenire della Corte europea dei diritti dell'uomo", in AIC, Rivista, 4/2012.

${ }^{53}$ Pto 5.3 del Considerato in diritto.
}

REVISTA DO DIREITO UNISC, SANTA CRUZ DO SUL №. 43 | p.03-24 | MAIO-AGO 2014 
prestazioni di diversi gruppi di contribuenti per come parametrato all'ammontare dei contributi effettivamente versati negli anni, determinandosi in un rischio di sostanziale disparità di trattamento a seconda del luogo di prestazione dell'attività lavorativa, laddove in concreto "i contributi versati in svizzera siano quattro volte inferiori a quelli versati in Italia" diventa così terreno di bilanciamento in concreto di valori costituzionali comunque omogenei tra loro. Ciò proprio al fine di garantire che i vantaggi invocati dai ricorrenti non rischino di risolversi in maggiori oneri per tutti gli altri contribuenti. Salvo che il legislatore non decida di intervenire con una soluzione sistemica che riequilibri il regime previdenziale riparametrando l'intero meccanismo di calcolo delle pensioni nel rispetto di una sostanziale equivalenza, però, tra contributi versati e prestazioni erogate tra tutti gli aventi diritto per categorie omogenee. Risultato non conseguibile ad opera di una sentenza di accoglimento della Corte costituzionale. Coerente sul piano del rispetto dei principi di eguaglianza e solidarietà, la sentenza lascia però impregiudicata la questione della irrisolta diversità di interpretazione, se non in ipotesi astratta, certamente nelle concrete applicazioni, dei limiti degli interventi legislativi con efficacia retroattiva ed effetti rilevanti nei giudizi in $\mathrm{corso}^{54}$. In attesa, forse, di una futura pronuncia "pilota" della Corte europea dei diritti dell'uomo o di un nuovo passo in avanti nel processo di integrazione del diritto costituzionale europeo dei diritti fondamentali da parte della Corte costituzionale italiana.

\footnotetext{
${ }^{54}$ Così, ancora, F. Gallo, Rapporti tra Corte costituzionale e Corte EDU, $9 \mathrm{~s}$.

REVISTA DO DIREITO UNISC, SANTA CRUZ DO SUL №. 43 | p.03-24 | MAIO-AGO 2014
} 